\title{
Norois
}

Environnement, aménagement, société

$241 \mid 2016$

Innovation sociale et développement des territoires dans les campagnes européennes

\section{Innover en territorialisant : quel est le prix à payer ? Analyse des coûts de transaction du programme LEADER 2007-2013 en Auvergne et Bourgogne}

Innovation and territorialization: how much to pay? An economic study of transaction costs applied to LEADER program 2007-2013 in two French regions (Auvergne and Burgundy)

Marielle Berriet-Solliec, Denis Lépicier et Dominique Vollet

\section{OpenEdition}

\section{Journals}

Édition électronique

URL : http://journals.openedition.org/norois/5981

DOI : $10.4000 /$ norois. 5981

ISBN : 978-2-7535-5504-4

ISSN : $1760-8546$

Éditeur

Presses universitaires de Rennes

Édition imprimée

Date de publication : 30 décembre 2016

Pagination : 15-32

ISBN : 978-2-7535-5483-2

ISSN : 0029-182X

Référence électronique

Marielle Berriet-Solliec, Denis Lépicier et Dominique Vollet, «Innover en territorialisant : quel est le prix à payer? Analyse des coûts de transaction du programme LEADER 2007-2013 en Auvergne et Bourgogne », Norois [En ligne], 241 | 2016, mis en ligne le 31 décembre 2018, consulté le 04 janvier 2020. URL : http://journals.openedition.org/norois/5981 ; DOI : 10.4000/norois.5981 


Presses
Universitaires
de Rennes
www.pur-editions.fr
Revue en ligne : http://norois.revues.org

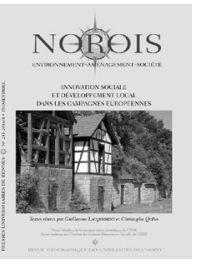

\title{
Innover en territorialisant : quel est le prix à payer? Analyse des coûts de transaction du programme LEADER 2007-2013 en Auvergne et Bourgogne
}

\author{
Innovation and Territorialization: How Much to Pay? \\ An Economic Study of Transaction Costs Applied to LEADER Program 2007-2013 \\ in Two French Regions (Awvergne and Burgundy)
}

\section{Marielle Berriet-Solliec ${ }^{* a}$, Denis LÉPICIER ${ }^{a}$ et Dominique Vollet ${ }^{b}$}

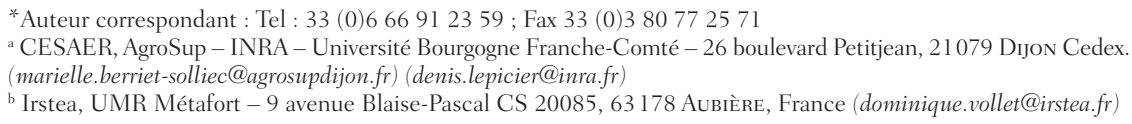

Résumé : Le caractère innovant du programme LEADER est analysé, dans cet article, à l'aune des avantages du processus de territorialisation par rapport à une politique descendante et de ses limites en termes de coûts de transaction. L'analyse est appliquée au programme LEADER 2007-2013 dans les régions Auvergne et Bourgogne, plus précisément à quatre projets de ces deux régions. L'estimation de ces coûts conclut à des valeurs élevées pour les porteurs de projet (notamment privés), en particulier en raison de la complexité du montage financier, mais pointe surtout le coût généré par l'ingénierie territoriale et la formation des parties prenantes qui sont identifiées comme deux facteurs essentiels pour la mise en œuvre du programme LEADER.

\begin{abstract}
This paper analyses the process of innovation in the LEADER program by assessing the advantages of the territorialization process with respect to a top-down policy and its limitations in terms of transaction costs. An empirical analysis of the 2007-2013 LEADER program is applied to two French regions: Awvergne (in south central France) and Burgundy (in east central France). The estimation of these costs highlights the difficulties faced by project leaders (especially private actors), in particular due to the complexity of the financial packages involved, but points above all to the costs generated by territorial engineering and the human capital of stakeholders, which are at the same time both recognized as key factors for promoting the territorialization process.
\end{abstract}

Mots clés : politique agricole, innovations territoriales, économie institutionnelle, gouvernance, LEADER

Keywords: agriculture policy, territorial innovations, institutional economics, governance, LEADER 


\section{INTRODUCTION}

Dans un contexte européen et national de restriction budgétaire, les exigences d'efficacité et d'efficience des politiques publiques sont aujourd'hui renforcées. Cette préconisation encourage les décideurs publics à repenser les fondements et les modalités des dispositifs d'intervention publique. Le recours aux procédures territorialisées visant à améliorer la pertinence des outils de l'action publique pour répondre aux enjeux spécifiques identifiés est souvent privilégié comme une forme innovante d'intervention, tant en termes organisationnels (gouvernance) qu'en termes d'originalité des projets soutenus. Dans cette perspective, le dispositif européen LEADER (Liaison entre les actions de développement de l'économie locale) est analysé à la fois comme un dispositif innovant d'intervention au regard de sa capacité à mobiliser conjointement des financements publics et privés autour d'une stratégie explicite de développement local (Thuesen et al., 2014), mais aussi comme une procédure qui active et révèle des stratégies inédites des acteurs locaux (Perrin, 2011; Dax et al., 2014; Sanche-Zamora et al., 2014). Ainsi, territorialiser un dispositif d'intervention publique apparaît comme une forme d'innovation dans la mesure où elle correspond non seulement à un changement d'échelle, mais implique également une approche intégrée du développement rural, une définition nouvelle des problèmes posés et un renouvellement des procédures de décision (Thuesen et al., 2014). Ainsi, territorialiser la politique de développement rural consiste à élargir le cadre sectoriel (au-delà du secteur agricole, souvent privilégié en zones rurales) mais aussi par une implication croissante des acteurs locaux qui expriment une diversité d'attentes et d'intérêts pas nécessairement convergents (et non une approche descendante qui serait l'apanage de décideurs nationaux) justifiant la mobilisation d'ingénierie de projet (Trouvé et al., 2007).

Pour les économistes, les avantages d'un mode d'intervention publique territorialisée sont toutefois discutés. Certes, les politiques territoriales permettraient une meilleure adaptation aux spécificités locales grâce à une valorisation des ressources spécifiques (Colletis-Wahl et Pecqueur, 2001) et une gestion plus efficace des interdépendances non marchandes (Berriet-Solliec et al., 2006). Pour autant, cette territorialisation de l'action publique est questionnée à l'aune des coûts de sa mise en œuvre (Irwin et al., 2010), supportés à la fois par le décideur public et les bénéficiaires (Kephaliacos et Robin, 2004), notamment pour les politiques plus innovantes (cf. le cas de Mesures agro-environnementales chez Rousset et Louis, 2012) ou encore pour les mesures agro-environnementales territorialisées pour lesquelles les effets propres sont limités (Chabé-Ferret et Subervie, 2013). Ces coûts sont appréhendés par les économistes par la notion de coûts de transaction, définie comme l'ensemble des coûts liés à la gestion, l'animation, le suivi d'une politique (Coase, 2005; Williamson, 1994) ${ }^{1}$.

Si de nombreux travaux examinent les effets attendus et observés des programmes LEADER ou des mesures agro-environnementales territorialisées en Europe (Dargan et Shucksmith, 2008; ValquiVidal, 2009; Chevalier et Dedeire, 2014, Maurel et al., 2014, Chabé-Ferret et Subervie, 2013), l'examen des modalités de conduite de ces politiques territoriales est beaucoup plus rare. Par exemple, Zahm et al. (2016) s'appuient sur une triangulation des approches pour mener une démarche d'évaluation intégrée de l'efficacité des Mesures AgroEnvironnementales Territorialisées (MAET) (évaluation des effets propres, évaluation coût-efficacité environnementale et évaluation du rôle des facteurs organisationnels dans le processus d'adhésion). Dans ce numéro spécial sur l'innovation territoriale, notre contribution d'économistes vise à alimenter le débat en proposant un éclairage particulier, celui des coûts de transaction, qui permet de questionner « le prix à payer » pour territorialiser et innover dans les interventions publiques dans les espaces ruraux.

À ce titre, le programme LEADER constitue un bel exemple pour tester cette approche. En effet, sa mise en œuvre s'appuie obligatoirement sur la mise en place d'un Groupe d'Action Locale (GAL) associant acteurs publics et privés au niveau de territoires de projet. Il est fréquemment l'objet de

\footnotetext{
1. Coase (2005) a défini le coût de transaction de la façon suivante : « Lorsque l'on souhaite opérer une transaction sur un marché, il est nécessaire de rechercher son ou ses contractants, de leur apporter certaines informations nécessaires et de poser les conditions du contrat, de conduire les négociations instaurant ainsi un véritable marché, de conclure le contrat, de mettre en place une structure de contrôle des prestations respectives des obligations des parties, etc. » Williamson les a formalisés de la façon suivante : "Les coûts de transaction constituent l'équivalent économique des frictions dans les systèmes physiques. L'économie des coûts de transaction pose le problème de l'organisation économique. »
} 
controverses, exprimées essentiellement par les financeurs, notamment en raison du coût élevé d'animation et de gestion qu'il implique (Cour des Comptes européenne, 2010; Balny et al., 2010). Les bénéfices ou les avantages attendus du processus de territorialisation sont souvent estimés de façon qualitative (Méasson, 2007), plus rarement de façon quantitative (Barbarot et al., 2013). Il peut s'agir de bénéfices marchands (valeur ajoutée supplémentaire conférée par une différenciation ou spécification des produits) mais aussi non marchands (renforcement des coordinations locales). Si les bénéfices sont étudiés, ils sont rarement mis en lien avec le coût supplémentaire que représentent les modalités d'animation particulières liées à la territorialisation du programme LEADER. Or, pour apprécier l'intérêt de ce type de dispositif et son caractère innovant, il est nécessaire de se doter d'un outil d'analyse (les coûts de transaction) pour mettre en regard les bénéfices attendus avec les coûts spécifiques de gestion et d'animation, afin de contribuer à une analyse de l'efficience des modes de gouvernance territoriale (Chia et al., 2008; Lardon et al., 2008). Dans une démarche d'évaluation, rappelons que l'efficience est définie comme le rapport entre les moyens mobilisés (matériels, humains, financiers) et les effets du dispositif d'intervention.

Cet article présente les résultats d'un travail appliqué au programme LEADER s'inscrivant dans la lignée des approches évaluatives de la gouvernance territoriale (Rey-Valette et Mathé, 2012). Souhaitant apporter une contribution économique à une analyse pluridisciplinaire du processus d'innovation du programme LEADER, cette contribution propose une estimation du niveau et de la variabilité des coûts de transaction propres au programme LEADER en les mettant en regard avec les bénéfices attendus des actions menées. Pour cela, nous avons estimé le niveau et les facteurs de variabilité affectant les coûts de transaction spécifiques à la mise en œuvre de ce programme. Cette méthode a été appliquée, sur la période 2007-2013, à un ensemble de projets LEADER dans deux régions françaises comparables du point de vue de l'importance des espaces ruraux dans le développement régional (l'Auvergne et la Bourgogne) mais différentes en ce qui concerne l'historique et la gestion des politiques territoriales (Berriet-Solliec et Chabé-Ferret, 2011).
Après avoir précisé notre grille d'analyse et la méthode d'investigation retenue, nous présenterons les résultats obtenus dans ces deux régions françaises.

\section{Cadre d'analyse ET ÉLÉMENTS DE MÉTHODE}

La question traitée repose sur deux dimensions, celle des bénéfices tirés de la territorialisation de la mise en œuvre du programme LEADER d'une part, et celle des coûts de transactions et de leur estimation d'autre part. Nous formulons l'hypothèse que ces deux dimensions sont interdépendantes. En premier lieu, pour un même niveau d'avantages tirés de la territorialisation, le niveau et la structure des coûts de transaction peuvent être très variables selon la nature juridique du porteur de projet et les caractéristiques du territoire. En second lieu, nous testons l'hypothèse d'une corrélation positive entre les coûts de transaction et le degré de territorialisation des projets, c'est-à-dire l'intensité des efforts d'adaptation du projet au contexte local et de mobilisation des acteurs.

\section{Méthode d'estimation des avantages de la territorialisation}

Les avantages, entendus comme les impacts positifs du programme LEADER ont d'ores et déjà été analysés, mais en retenant des aspects particuliers : la capacité à articuler des logiques contradictoires (Méasson, 2007), la satisfaction des besoins locaux (Cour des comptes européenne, 2010) ou encore l'augmentation des capacités locales (Commission européenne, 2010). En raison de la difficulté à gérer la diversité des objectifs poursuivis par le programme LEADER (Mathé et al., 2014; Chevalier et Dedeire, 2014), nous avons opté pour une approche élargie qui intègre la diversité des avantages de ce programme, en ayant le souci de proposer une méthode systématique de quantification des avantages de la territorialisation adaptable à la diversité des projets : projets publics et privés, aussi bien de taille réduite que d'une certaine ampleur (allant de la rénovation d'un commerce à l'établissement d'un complexe touristique).

Cette évaluation du degré de territorialisation a été réalisée à l'échelle de 18 projets LEADER 
2007-2013 dans les régions Auvergne et Bourgogne selon une approche quantitative mobilisant les données socio-économiques principalement issues de l'Insee et les données financières relatives aux projets financés dans les programmes LEADER à partir de la base de données OSIRIS. Cette base, gérée par l'Agence de Services et de Paiements (ASP) ${ }^{2}$, regroupe toutes les informations relatives à l'instruction des dossiers LEADER.

Pour estimer le degré de la territorialisation de ces 18 projets LEADER, nous avons retenu quatre mécanismes de territorialisation des projets identifiés initialement par Michel (2010), repris par Gloria (2011) et affinés par Barbarot et al. (2013) :

- l'ancrage territorial : capacité des porteurs de projet à prendre en compte les enjeux locaux et les ressources territoriales,

- l'intersectorialité : capacité des acteurs professionnels de secteurs différents à se mobiliser dans des projets communs,

- la coordination entre acteurs : capacité des acteurs à créer ou s'intégrer dans une dynamique de coopération dans le cadre de projets de développement territorial,

- la transversalité : capacité d'un projet à prendre en compte simultanément les différentes dimensions du développement durable.
À ces quatre mécanismes identifiés par Barbarot et al., 2013 sont associés des critères précis que nous avons conçus pour caractériser les projets financés par les programmes LEADER et qui ont constitué la grille d'analyse des projets (figure 1). Au total, chaque projet est noté sur la base des réponses à 11 questions associées aux critères de territorialisation, la note maximale étant de 13 (voir annexe 1).

La grille d'analyse a été remplie après analyse des dossiers de demande de financements ainsi que d'un entretien auprès de personnes ayant assuré la maîtrise d'ouvrage. Cet entretien a été réalisé sur la base d'un guide semi-directif structuré en plusieurs parties : historique de la structure, insertion dans les réseaux techniques, économiques, sociaux; genèse du projet et mise en ouvre, mobilisation de compétences extérieures, des partenaires financiers, les résultats observés ou attendus.

\section{Une grille d'analyse des déterminants des coûts de transaction}

Le champ d'application de la théorie des coûts de transaction (TCT) est celui de l'économie de marché; il a été proposé par des auteurs comme Dahlman (1974) puis développé par Williamson (1994) ou encore Coase $(2005)^{3}$. Toutefois, de

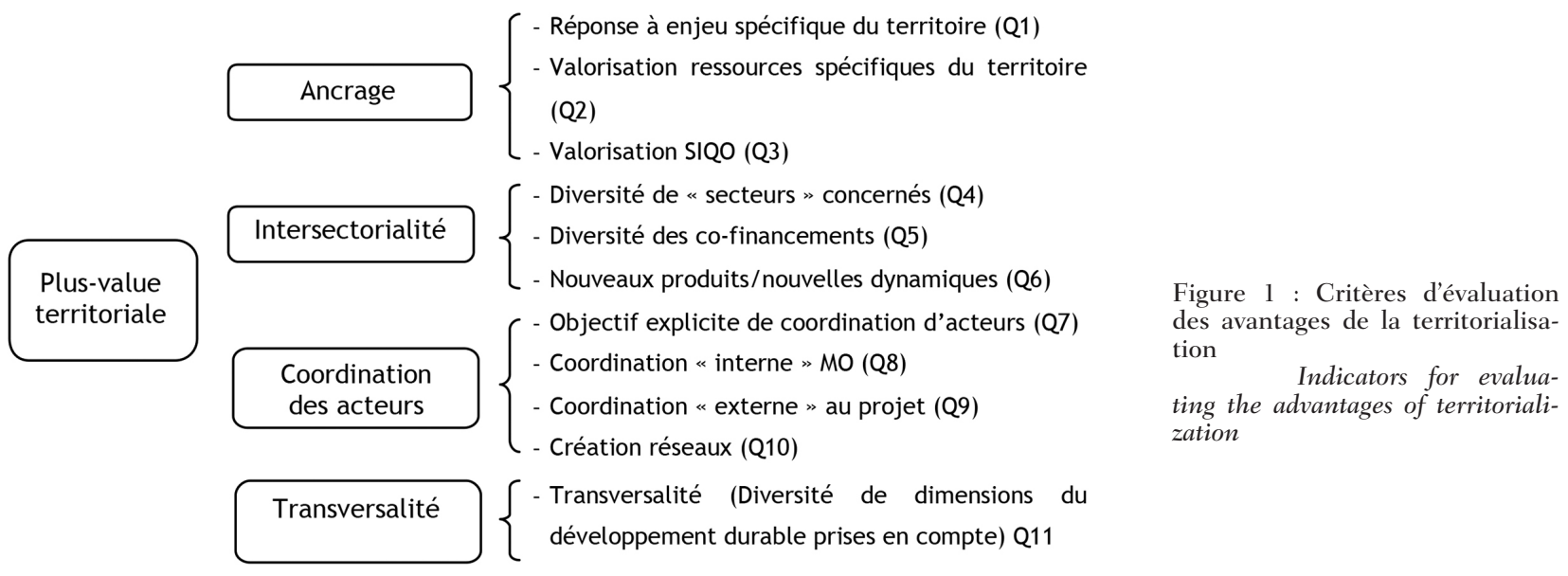

2. Cette base administrative est alimentée par les différents organismes participant à la mise en œuvre des programmes LEADER : Direction Départementale des Territoires (DDT), Direction Régionale de l'Agriculture, de l'Alimentation et de la Forêt (DRAAF), Groupes d'Action Locale.

3. Dahlman (1974) classe les coûts de transaction en 3 catégories : coûts de recherche et d'information » : prospection, comparaison du rapport qualité/prix des différentes prestations proposées, étude de marché etc.

- « coûts de négociation et de décision » : rédaction et conclusion d’un contrat etc.

- « coûts de surveillance et d'exécution » : contrôle de la qualité de la prestation, vérification de la livraison, etc. 
nombreux économistes considèrent que ce cadre d'analyse peut être mobilisé pour l'étude des coûts de transaction associés aux politiques publiques (Mac Cann et al., 2005), en particulier lorsqu'elles concernent l'environnement ou plus largement le développement rural. Pour tenir compte des coûts de transaction liés aux politiques publiques, il s'agit de reconsidérer la définition de ces coûts, telle qu'elle a été établie pour analyser les échanges marchands. Tandis que Ménard (2003) définit la transaction comme étant un « transfert entre unités technologiquement séparables de droits d'usages sur les biens et services ", la définition proposée par Mac Cann (2005) qui cible « les coûts des ressources utilisées pour définir, établir, maintenir et échanger les droits de propriété » semble plus adaptée pour appréhender les coûts d'information, de gestion administrative et d'animation en jeu dans les politiques de développement rural. À partir de l'évaluation des coûts de transaction que supporte une entreprise, ce cadre théorique rend compte de la structure de gouvernance que celle-ci va adopter. Nous nous proposons de le retenir dans le cadre d'une démarche évaluative de politiques publiques territoriales, non pas pour déterminer la structure de gouvernance la plus appropriée, mais comme grille d'analyse de la nature et de la variabilité des coûts de transaction. Dans le cas d'un projet soutenu par le programme LEADER, on peut parler de «transaction » entre l'Union Européenne et le porteur de projet via un groupe d'action locale (GAL) générant d'une part, des coûts financiers directs, tels que le versement d'une subvention aux porteurs de projet ou l'embauche d'agents de développement pour conduire l'animation du programme et d'autre part, des coûts liés au temps de travail administratif engagé par les opérateurs publics concernés. Ces coûts sont à apprécier en rapport avec les effets positifs (les avantages) sur le développement rural produits par l'action relative à ces coûts.

L'exposé des principes de mise en œuvre des programmes LEADER permet d'envisager la nature des transactions en jeu. Les programmes LEADER sont ainsi définis à un niveau infrarégional par des GAL qui regroupent différents acteurs (profession agricole, touristique, associations, chambres consulaires, élus locaux), intervenant dans le développement rural du territoire autour desquels s'établit le GAL. En France, les GAL se constituent obligatoi- rement à l'échelle de territoires de projet existants (le plus souvent, Pays, PNR) et sont portés généralement par des syndicats mixtes ou des associations. Les GAL sont retenus dans le cadre d'une démarche de sélection à l'échelle régionale sur la base de la pertinence de la stratégie et de la gouvernance proposées par les territoires candidats. Si celle-ci est acceptée, à l'issue du processus de sélection conduit au niveau régional, l'équipe d'animation accompagne, voire démarche des porteurs de projet concourant à la réalisation de la stratégie du GAL en leur proposant des incitations financières et de l'expertise de montage de projets prévus dans le programme LEADER. Ceux-ci doivent alors constituer un dossier de demande de subvention qui est examiné par le Comité de Programmation du GAL, lequel décide après délibération d'accorder ou non une subvention au porteur de projet. L'attribution d'une subvention enclenche une procédure administrative engageant, dans la période 2007-2013, les services de l'État à l'échelle départementale (Direction départementale des territoires - DDT) ou régionale (Direction régionale de l'agriculture, de l'alimentation et de la forêt - DRAAF) pour vérifier l'éligibilité au programme national et européen dans lequel s'insère le programme LEADER; pour recueillir les justificatifs administratifs attestant du bon usage des financements; enfin un contrôle par l'Agence de Services et de Paiements (ASP) pouvant également être effectué (figure 2).

Différents types d'acteurs peuvent engendrer des coûts de transaction : l'équipe d'animation LEADER (qui accompagne le porteur de projet pour constituer le dossier, la demande de paiement), le service référent (qui instruit le dossier, transmet la demande de paiement, produit certificat de service fait), le comité de programmation du GAL qui émet une décision, l'organisme payeur (qui verse l'aide).

Cette analyse des types de transaction en jeu dans le cadre des programmes LEADER, a permis de construire une grille d'analyse des coûts de transaction en la structurant autour des trois attributs que la théorie des coûts de transaction retient classiquement pour expliquer l'importance des transactions : i) la spécificité des actifs, ii) l'incertitude et iii) la fréquence des transactions (Williamson, 1994).

La spécificité des actifs renvoie à l'idée selon laquelle, plus la transaction concerne un objet singulier, nouveau et très lié au contexte local dans lequel 


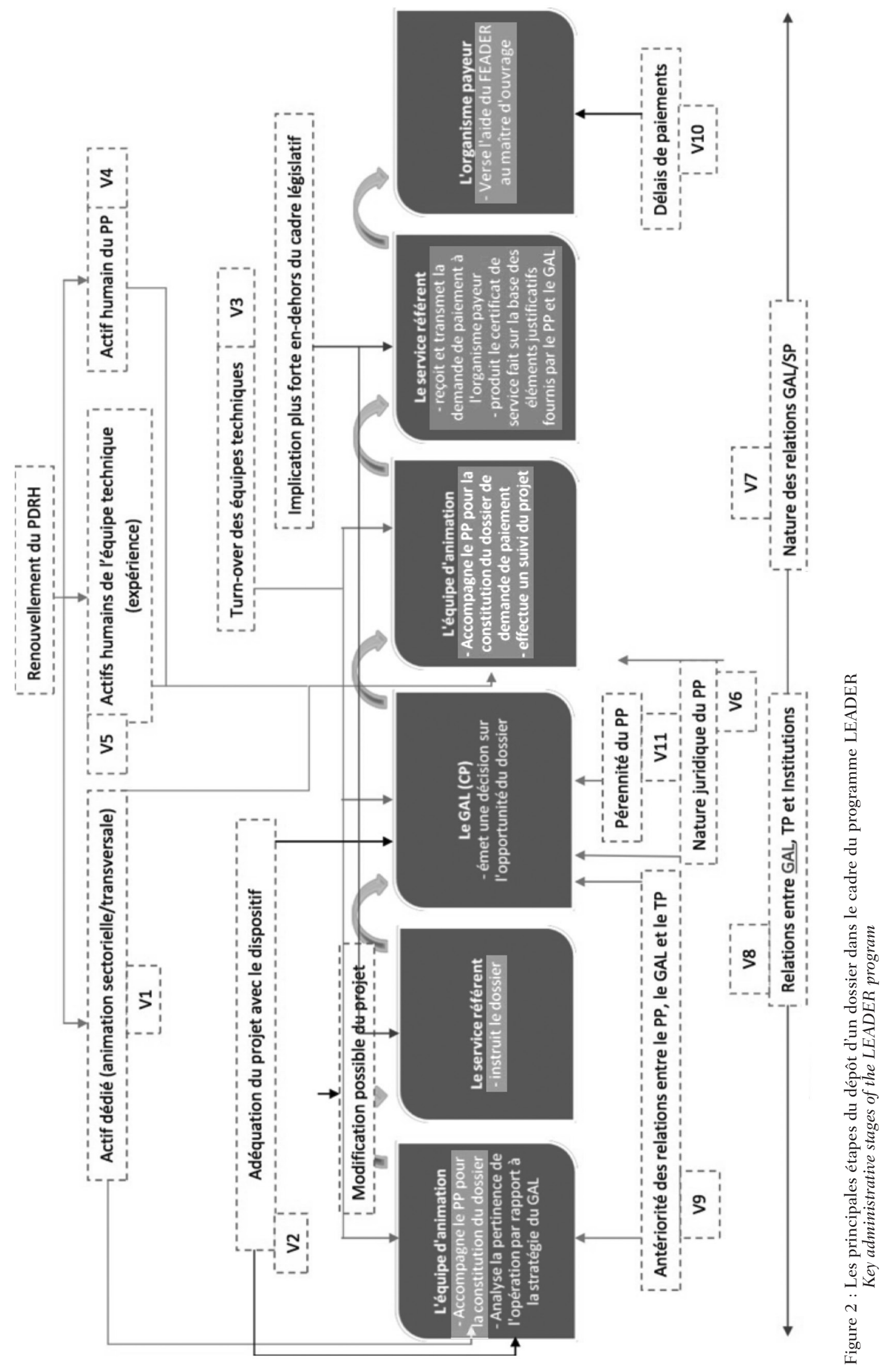


la transaction est réalisée, plus le coût de la transaction tend à être élevé. L'incertitude renvoie quant à elle aux efforts à consentir pour accéder à l'information et pour réduire la prise de risque. Enfin, la fréquence repose sur l'hypothèse que des effets d'apprentissage peuvent résulter d'une occurrence plus élevée d'une situation de transaction. Selon cette hypothèse, ce dernier attribut peut modifier les conséquences des deux premiers sur le coût de transactions, c'est pourquoi le cadre d'analyse des coûts de transaction adapté à notre objet s'articule autour des deux premières dimensions. Nous avons identifié 11 déterminants (et 10 variables explicatives) des coûts de transaction, 6 portant sur la spécificité des actifs et 5 sur l'incertitude (figure 3).

Le niveau de spécificité des actifs dépend:

- du type d'animation (variable V1) : les coûts de transaction supportés par les territoires de projet seront importants dans le cas où il existe du personnel dédié à une animation locale et transversale; - de l'adéquation du projet avec le dispositif de financement (variable V2) : pour tous les partenaires, un projet correspondant au dispositif de soutien public et à son territoire demandera moins de modifications et donc moins de coûts de transaction;

- du turn-over des équipes d'animation (variable V3) : un renouvellement important des équipes techniques entre chaque programmation ou plus encore pendant la programmation pourra expliquer un niveau élevé de coût de transaction;
- de l'expérience des porteurs de projet (variable V4) : des porteurs de projets bien formés, insérés dans des réseaux, ayant une expérience dans le montage de dossiers supporteront moins de coûts de transaction;

- de l'expérience des membres de l'équipe d'animation (variable V5) : un chargé d'animation et un gestionnaire habitués au montage de dossiers LEADER passeront moins de temps et donneront des conseils plus avisés aux porteurs de projet.

- de la nature juridique du porteur de projet (variable V6) : un porteur de projet public familier des règles de gestion, bénéficiant d'un suivi informel du dossier supportera moins de coûts de transaction qu'un porteur de projet privé ou associatif peu rompu à ce type d'exercice et peu familier des règles de gestion.

Cinq déterminants relatifs à l'incertitude ont été repérés :

- la nature des relations entre GAL et territoires de projet (variable $V 7$ ) : les coûts de transaction sont d'autant plus élevés que les animations se font en parallèle et que la complémentarité entre équipes techniques est faible;

- la nature des relations entre GAL, services de l'État et territoires de projet (variable V8) : dans certains cas, les relations peuvent être tendues, notamment en raison d'un partage des tâches confus accroissant ainsi les coûts de transaction;

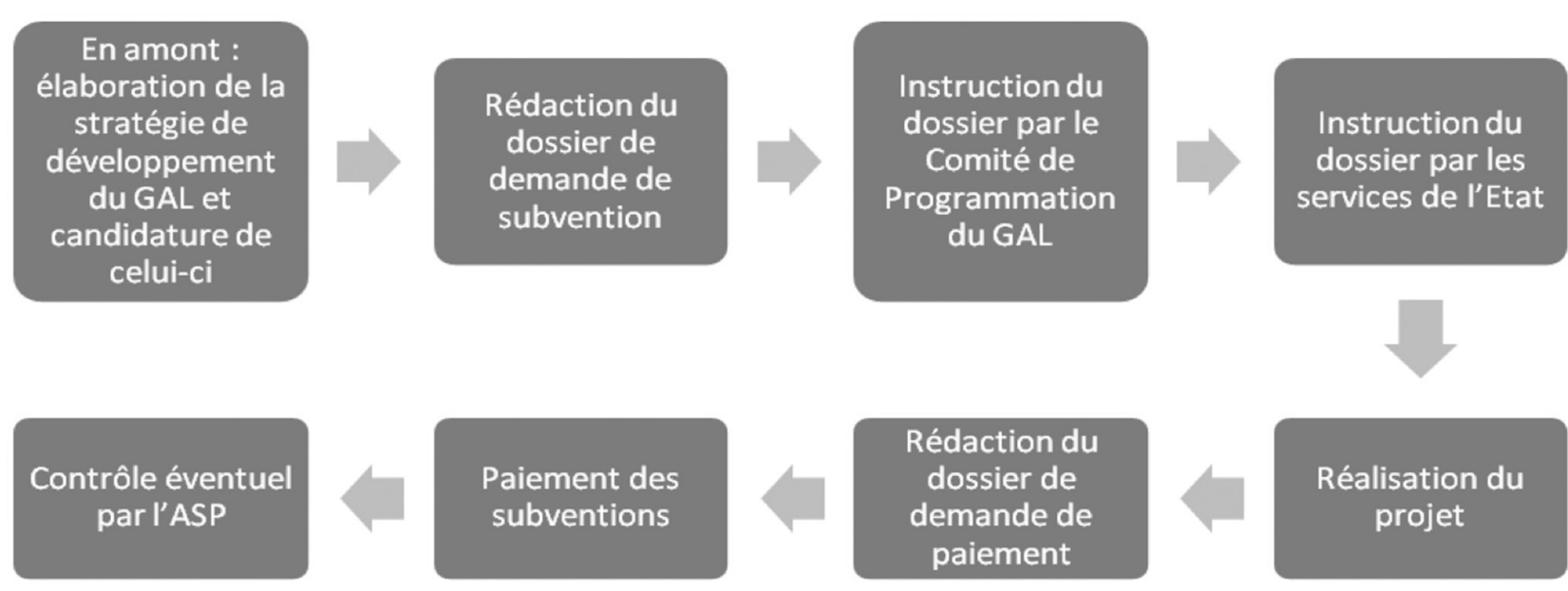

Figure 3 : Schéma récapitulatif des caractéristiques du programme LEADER génératrices de coûts de transaction suivant les différentes étapes de gestion du dispositif tation

Diagram summarizing the various characteristics of the LEADER program that generate transaction costs at each stage of implemen- 
- l'antériorité des relations entre porteurs de projet, GAL et territoires de projet (variable V9) : les coûts de transaction sont d'autant plus élevés que les relations sont récentes et la confiance faible;

- les délais de paiement (variable V10) : pour tous les partenaires, lincertitude concernant les délais de paiement peut être importante;

- la pérennité du porteur de projet (variable V11): l'incertitude concernant la pérennité du porteur de projet est nulle pour certains porteurs de projets (publics) mais très variable pour les porteurs associatifs ou privés.

Les déterminants précédemment identifiés ont été estimés par le biais de questions spécifiques (voir annexe 2). Cette évaluation a été faite précisément pour quatre projets parmi les 18 analysés initialement au regard de leur degré de territorialisation (voir annexe 3, tableaux 1 et 2). Pour ces quatre projets LEADER, dont les critères de sélection seront précisés dans la partie 2.1 de cet article, une évaluation en deux temps est effectuée pour évaluer le niveau des coûts de transaction et leur répartition entre les acteurs concernés.

Premièrement, une évaluation estime le coût « effectif » supporté par les différents acteurs. Pour le porteur de projet, le temps passé pour monter son projet auprès des différentes administrations ainsi que le temps consacré aux démarches administratives pendant et après la mise en place effective du projet ont été estimés. Pour l'équipe d'animation, le temps consacré à chaque projet a également été estimé.

Deuxièmement, il est procédé à une estimation des déterminants identifiés comme faisant varier les coûts de transaction qui sont supportés par le porteur de projet et le GAL ou le territoire de projet par enquête auprès des porteurs de projets, des services techniques du GAL et des services instructeurs pour une vingtaine de projets des régions Auvergne et Bourgogne. Pour chacune des variables, une appréciation, placée sur une échelle de 1 à 7 , est exprimée soit par le porteur de projet soit par l'équipe chargée d'animation (selon le déterminant) (voir annexe 2, colonne 2). La somme des valeurs attribuées selon les échelles de Lickert ${ }^{4}$ a ensuite

4. Une échelle de Likert représente une échelle de jugement grâce à laquelle une personne interrogée fait part de son degré d'accord ou de désaccord vis-à-vis d'une affirmation (l'énoncé). L'échelle est constituée en général de cinq ou sept choix de réponse qui permettent de nuancer le degré fourni des estimations des niveaux de coûts de transactions supportés par les animateurs et les porteurs de projet. De plus, les enquêtes ont été l'occasion d'appréhender les conditions de mise en œuvre du programme Leader (voir annexe 2, colonne 3), afin d'améliorer les estimations et leurs interprétations fournies.

Les estimations des coûts de transaction «effectifs » (estimés en heures dans nos enquêtes auprès des porteurs de projets et des équipes d'animation) sont données à titre indicatif pour appréhender leur ordre de grandeur entre les projets et par rapport aux soutiens publics accordés. En effet, concernant l'estimation des coûts de transaction dans les politiques agricoles et de développement rural, l'OCDE (2007) mentionne que « les réponses aux questionnaires et entretiens directs sont une source de distorsion car les personnes ont tendance à surestimer leur efficacité et à sous-estimer les CTLP [coûts de transaction liés aux politiques]. Inversement, les gestionnaires pourraient surestimer les CTLP pour obtenir davantage de ressources ». Pour cette raison, l'essentiel des commentaires est basé sur l'estimation par échelle de Lickert des principaux déterminants des coûts de transaction.

\section{Principaux Résultats}

\section{Le choix de quatre projets au degré de territorialisation élevé}

Compte tenu de la quantité d'informations nécessaires pour évaluer finement les coûts de transaction et leurs déterminants, les estimations ont été conduites sur quatre projets pour mettre en évidence la variabilité du niveau et de la structure des coûts de transaction selon la nature juridique du porteur de projet et les caractéristiques du contexte territorial (tableau 1). Plusieurs critères justifient le choix des projets. Tout d'abord le choix de projets présentant des degrés élevés de territorialisation en raison de la mise en réseau d'acteurs et d'innovation visait à centrer l'analyse sur les déterminants des coûts de transaction et non sur ceux du degré de territorialisation. Ensuite, les projets couvrent les différents types de porteurs de projets (porteurs de projet publics, associatifs et privés) du fait de

d'accord. Nous avons retenu ici une échelle impaire où le niveau central (le chiffre 4 dans celle retenue de 7 ) permet de n'exprimer aucun avis. 
l'hypothèse émise sur l'influence forte de ce paramètre dans la constitution des coûts de transaction (annexe 3 : tableau 1). Parmi les quatre projets retenus, deux projets concernent une thématique agricole : la communication d'un collectif d'agriculteurs vendant leurs produits en circuit court et la création d'un caveau collectif d'une AOC viticole. Deux concernent le tourisme et plus largement le développement rural : (1) un chantier de restauration, d'aménagement et de valorisation touristique d'un château par l'action commune de deux municipalités et d'une association (2) l'appui à l'installation d'une entreprise d'aide à la création d'entreprise.

Selon la méthode d'estimation des avantages de la territorialisation présentée précédemment et dont les résultats sont détaillés dans le tableau 2, les quatre projets se caractérisent par un degré de territorialisation élevé par rapport aux autres projets LEADER étudiés (moyenne de 8,2; note minimale de 1 et maximale de 12). Les projets à thématique agricole (projet 1 sur la communication d'un collectif d'agriculteurs et projet 2 de création d'un caveau collectif) enregistrent des degrés de territorialisation plus faibles que les projets relevant des autres thématiques (note de 9 pour les projets 1 et 2) car ils restent limités à la sphère agricole. Le score relatif à l'intersectorialité est particulièrement faible pour le projet 2 (car concernant les seuls agriculteurs de la cave). Pour le projet 1 , c'est la coordination entre acteurs qui est plus faible en raison d'une dynamique territoriale envisagée de façon descendante dans le département de la Haute-Loire sous la pression du Conseil général. Il en résulte que « les GAL sont beaucoup moins dynamiques sur leur territoire par manque de soutien et ils ne disposent pas de moyens humains suffisants pour assurer l'animation sur leur territoire ou ne s'en donnent pas les moyens » (entretien auprès d'un chargé de mission). Ce résultat est observé pour l'ensemble des projets relevant de cette thématique.

Les projets à vocation de développement rural (projet 3 à vocation d'accueil dans un château et projet 4 d'un établissement d'appui aux créateurs) ont un degré de territorialisation plus marqué (notes de 12 et 10 respectivement pour les projets 3 et 4 ) qui s'explique notamment par les meilleurs scores obtenus sur les critères de l'intersectorialité et de la coordination entre acteurs. Pour ces deux projets, le programme LEADER « a permis d'allier les envies et savoirs faire de $\mathrm{M}^{\mathrm{me}} \mathrm{X}$ (entrepreneuse et directrice d'une coopérative d'activités et d'emplois) et les compétences et les réseaux de Y (chargé de mission LEADER et des politiques) ». La remontée des projets est particulièrement efficace sur le ter-

\begin{tabular}{|c|c|c|c|c|}
\hline & \multicolumn{2}{|c|}{ Projets LEADER ayant une thématique agricole } & \multicolumn{2}{|c|}{$\begin{array}{l}\text { Projets LEADER ayant une thématique tourisme- } \\
\text { développement rural }\end{array}$} \\
\hline Nature du projet & $\begin{array}{l}\text { Communication d'un } \\
\text { collectif d'agriculteurs } \\
\text { (projet l ou Al de } \\
\text { l'annexe 3) }\end{array}$ & $\begin{array}{c}\text { Création d'un caveau } \\
\text { collectif d'une AOC } \\
\text { viticole } \\
\text { (projet } 2 \text { ou A2 de } \\
\text { l'annexe 3) }\end{array}$ & $\begin{array}{c}\text { Développement d'une } \\
\text { offre d'accueil (jeunes) } \\
\text { de qualité dans un } \\
\text { château } \\
\text { (projet } 3 \text { ou Pul de } \\
\text { l'annexe 3) }\end{array}$ & $\begin{array}{l}\text { Création d'un établis- } \\
\text { sement d'appui aux } \\
\text { créateurs d'entreprises } \\
\text { (projet } 4 \text { ou Prl de } \\
\text { l'annexe 3) }\end{array}$ \\
\hline $\begin{array}{l}\text { Maître d'ouvrage (por- } \\
\text { teur de projet) }\end{array}$ & $\begin{array}{l}\text { Association d'agricul- } \\
\text { teurs }\end{array}$ & $\begin{array}{l}\text { Organisme de Défense } \\
\text { et de Gestion de l'AOC } \\
\text { viticole }\end{array}$ & $\begin{array}{l}\text { Communauté de Com- } \\
\text { munes }\end{array}$ & SARL \\
\hline Localisation & Haute-Loire & Saône-et-Loire & Allier & Allier \\
\hline $\begin{array}{l}\text { Montant total de l'inves- } \\
\text { tissement }(€)\end{array}$ & 21000 & 175000 & 130000 & 64000 \\
\hline Aides publiques $(€)$ & 10860 & 92000 & 28000 & 45000 \\
\hline dont LEADER $(€)$ & 6333 & 50000 & 28000 & 14000 \\
\hline $\begin{array}{l}\text { Nombre de bénéfi- } \\
\text { ciaires } \\
\text { directs concernés* }\end{array}$ & 13 & 35 & - & - \\
\hline
\end{tabular}

Tableau 1 : Présentation des 4 études de cas Presentation of the four case studies 


\begin{tabular}{|l|c|c|c|c|c|}
\hline & Ancrage territorial & Intersectorialité & $\begin{array}{c}\text { Coordinations entre } \\
\text { acteurs }\end{array}$ & Transversalité & Total (sur 13) \\
\hline Projet 1 & 2 & 3 & 2 & 2 & 9 \\
\hline Projet 2 & 2 & 2 & 3 & 3 & 9 \\
\hline Projet 3 & 2 & 3 & 4 & 2 & 12 \\
\hline Projet 4 & 2 & 3 & 3 & 3 \\
\hline
\end{tabular}

Tableau 2 : Le degré de territorialisation des projets étudiés (source : traitements par les auteurs) Degree of territorialization in the projects studied

ritoire de projet en Sologne et Bocage Bourbonnais en raison d'une « bonne implication historique des DDT et de la préfecture » (entretien avec un acteur du territoire).

\section{Des niveaux et structures des coûts de transaction contrastés}

\section{Quels déterminants des coûts supportés par les porteurs de projet?}

Les déterminants des coûts supportés par les porteurs de projet sont de deux ordres : la nature juridique du porteur et le type d'aide apportée par les animateurs.

La nature juridique du porteur de projet : une influence majeure sur les coûts de transaction supportés par les porteurs de projets

L'un des résultats marquants est l'influence majeure de la nature juridique du porteur de projet sur les coûts de transaction qu'il supporte. En ce qui concerne les coûts liés à la spécificité des actifs, les deux projets portés par des acteurs privés ou asso- ciatifs (projets 1 et 2) sont proches de 40 alors qu'ils sont deux fois plus faibles pour les projets portés par des acteurs publics (projets 3 et 4). L'ordre de grandeur est également du simple au double pour les coûts liés à l'incertitude (d'un niveau proche de 10 pour les projets 1 et 2 et de 4 pour le projet 3 ). Les estimations en termes de temps passé vont dans le même sens que les résultats ci-dessus : en effet, pour les deux projets agricoles, le temps passé par les porteurs de projets associatifs sans lien avec le décideur public (projets 1 et 2) représente environ $75 \%$ du temps total alors que la proportion est de l'ordre de la moitié pour les deux autres (tableau 3).

Plusieurs éléments peuvent expliquer les différences de coûts de transaction supportés par les porteurs de projets publics et privés. D’abord, le porteur de projet privé n'est pas familier des règles de comptabilité et de gestion administrative des fonds publics, au contraire des porteurs de projet publics, ce qui explique des difficultés plus importantes rencontrées dans le remplissage des dossiers de demande de subvention et de paiement. Les porteurs de projet publics ont quant à eux indiqué lors des questions ouvertes qu'ils considéraient la recherche de financements comme faisant partie

\begin{tabular}{|c|c|c|c|c|c|c|}
\hline & \multicolumn{3}{|c|}{$\begin{array}{l}\text { Estimation des principaux déterminants des coûts de transac- } \\
\text { tion par échelle de Lickert }\end{array}$} & \multicolumn{3}{|c|}{$\begin{array}{l}\text { Coûts de transaction « effectifs » } \\
\quad \text { (temps passé en heures) }\end{array}$} \\
\hline & \multirow{2}{*}{$\begin{array}{l}\text { Coûts de transac- } \\
\text { tion supportés par } \\
\text { les animateurs }\end{array}$} & \multicolumn{2}{|c|}{$\begin{array}{l}\text { Coûts de transaction supportés par les } \\
\text { porteurs de projet }\end{array}$} & \multirow{2}{*}{$\begin{array}{l}\text { Par l'équipe } \\
\text { d'animation }\end{array}$} & \multirow{2}{*}{$\begin{array}{l}\text { Par le porteur } \\
\text { de projet }\end{array}$} & \multirow{2}{*}{ Total } \\
\hline & & $\begin{array}{l}\text { liés à la spécificité } \\
\text { des actifs (V1 à V5) }\end{array}$ & $\begin{array}{l}\text { Liés à l'incertitude } \\
\text { (V7 à V11) }\end{array}$ & & & \\
\hline Projet 1 & 20 & 39 & 8 & 248 & $744(75 \%)$ & 992 \\
\hline Projet 2 & 26,5 & 39 & 11 & 120 & $432(78 \%)$ & 552 \\
\hline Projet 3 & 12,5 & 17,5 & 4 & 50 & $60(54 \%)$ & 110 \\
\hline Projet 4 & 27 & 18 & 18,5 & 248 & $250(50 \%)$ & 498 \\
\hline
\end{tabular}

Tableau 3 : Estimation des coûts de transaction dans les 4 études de cas (source : traitements par les auteurs) Estimation of transaction costs in the four case studies 
intégrante de leur métier. Ils disposent la plupart du temps de secrétaires compétentes dans la gestion administrative. Ensuite, la recherche de cofinancement (obligatoire pour la programmation LEADER 2007-2013) rend encore plus complexe et plus incertaine l'obtention d'un soutien public pour un porteur privé peu habitué à l'ingénierie financière publique.

Enfin, il convient de noter le cas particulier du projet 4. Le porteur de projet est une SARL jouant un rôle d'aide à la création d'entreprises. Elle est financée à hauteur de $50 \%$ par le Conseil régional d'Auvergne et est implantée dans le Puy de Dôme. Le Conseil régional souhaitant qu'elle puisse être accessible aux entrepreneurs des autres départements auvergnats, elle a incité l'entreprise à s'installer sur le territoire d'un GAL de l'Allier. Ce porteur de projet privé très en lien avec les acteurs publics supporte un niveau de coûts de transaction liés à la spécificité des actifs comparable à un porteur de projet public mais les coûts liés à l'incertitude sont d'un niveau élevé $(18,5)$, même pour un porteur privé, en raison de problèmes administratifs très particuliers ${ }^{5}$.

\section{Une influence déterminante de l'aide apportée par les animateurs pour réduire les coûts de transaction supportés par les porteurs de projets}

Les études de cas ont confirmé le rôle important des chargés de mission et animateurs des communautés de communes et des Pays dans l'accompagnement des projets financés par les programmes
LEADER : recherche de cofinancements, montage financier du projet (chaque cofinancement ne pouvant intervenir que dans certaines proportions, définies dans chaque dispositif du programme de développement rural), appui au montage du dossier et à la demande de paiement, les deux premières tâches étant systématiques.

L'estimation quantitative de l'aide apportée par les animateurs enquêtés est décomposée en cinq types de tâches: l'aide apportée à la recherche de cofinancements, au montage financier du projet, à l'élaboration du dossier de demande de paiement, et enfin à l'élaboration du dossier de demande de paiement. La cinquième tâche a été introduite pour pouvoir prendre en compte l'aide supplémentaire parfois fournie par l'animateur, et non comptabilisable dans les parties précédentes. En fonction des propos recueillis lors de la phase d'enquête, nous avons attribué une appréciation qualitative à chacune de ces parties : 0 (absente), 1 (présente) ou 2 (très poussée). La somme des notes attribuées à chaque tâche conduit à une note globale de l'aide apportée par le GAL (tableau 4).

Les différences apparaissent dans l'intensité de l'aide fournie dans le remplissage des dossiers administratifs, certains se contentant d'expliciter au porteur de projet les consignes de remplissage, d'autres poussant le soutien jusqu'à le remplir à leur place. Les différences concernent également l'aide apportée pour l'élaboration du dossier de demande de paiement, qui n'est pas toujours effectuée, l'animateur renvoyant généralement dans ce cas le porteur de projet au gestionnaire du programme.

\begin{tabular}{|l|c|c|c|c|c|c|c|}
\cline { 2 - 9 } \multicolumn{1}{c|}{} & $\begin{array}{c}\text { Co-finance- } \\
\text { ments }\end{array}$ & $\begin{array}{c}\text { Montage } \\
\text { financier }\end{array}$ & $\begin{array}{c}\text { Aide remplis- } \\
\text { sage dossier } \\
\text { subvention }\end{array}$ & $\begin{array}{c}\text { Aide remplis- } \\
\text { sage dossier } \\
\text { paiement }\end{array}$ & Aide sup. & $\begin{array}{c}\text { Coûts de } \\
\text { transaction } \\
\text { liés à l'incerti- } \\
\text { tude }\end{array}$ \\
\hline Projet 3 & 1 & 1 & 2 & 2 & 1 & 7 & 4 \\
\hline Projet 1 & 1 & 1 & 1 & 1 & 1 & 5 & 8 \\
\hline Projet 4 & 1 & 1 & 1 & 0 & 0 & 3 & 11 \\
\hline Projet 2 & 1 & 1 & 0 & 0 & 3 & 18,5 \\
\hline
\end{tabular}

5. La subvention LEADER ayant été accordée sur la base du temps que le porteur de projet consacrait à sa nouvelle activité, celui-ci a dû justifier toutes les heures passées, y compris entretiens téléphoniques et courriels pour pouvoir bénéficier de la somme globale qui lui avait été accordée, sans que l'animateur soutienne le porteur de projet dans cette tâche. En définitive, le montant des subventions a même été diminué, le porteur de projet n'ayant pas réussi pas à fournir tous les justificatifs nécessaires.
Tableau 4 : Appréciation de l'aide apportée par les animateurs aux porteurs de projet (source : traitement des auteurs)

to project leaders 
Deux territoires de projet ont fourni une aide plus importante :

- le GAL du projet 3, dont l'animatrice apporte une aide significative au porteur de projet, allant même parfois jusqu'à «tenir le stylo » dans le cas où le porteur de projet n'arrive pas à expliciter de manière claire les objectifs de son projet;

- le GAL du projet 1, où l'animatrice s'est beaucoup investie dans le projet car c'était l'un des premiers projets soutenus par ce GAL.

Dans nos quatre études de cas, il apparaît un lien fort entre le niveau d'incertitude auquel doit faire face le porteur de projet et le niveau d'aide apportée par l'équipe d'animation. Le projet 1 et surtout le projet 3 ont des niveaux limités de coûts de transaction, qui sont eux-mêmes liés à l'incertitude jugée comme faible (respectivement 8 et 4 ) en raison du soutien appuyé des animateurs et gestionnaires du GAL. Inversement, dans le cas où le soutien est plus léger et ne s'étend pas au remplissage du dossier de paiement, même dans le cas d'un dossier sans difficulté particulière (cas du projet 4), le niveau d'incertitude est plus élevée que précédemment (11), voire très élevé en cas de problèmes spécifiques (comme cela a été le cas pour le projet 4 , qui a rencontré des obstacles juridiques pour la création d'entreprise).

\section{Des coûts de transaction supportés par les équipes d'animation très sensibles au contexte territorial}

Deux ensembles de facteurs ont été identifiés comme déterminants des coûts supportés par les structures d'animation: les apprentissages personnels ou institutionnels par rapport aux dispositifs (expérience de l'animateur notamment) et les coordinations entre co-financeurs (relations entre équipes d'animation des programmes tout particulièrement).

\section{Des phénomènes d'apprentissage déterminants pour expliquer le niveau des coûts de transac- tion supportés par les équipes d'animation}

L'expérience de l'animateur a un rôle primordial dans le niveau de coût de transaction supporté par les structures d'animation. La quasi-totalité des animateurs rencontrés a souligné leur difficulté à intégrer les démarches LEADER. Les apprentissages de ces dispositifs sont effectivement très importants : le cadre du PDRH est très contraignant, tant au niveau de la conditionnalité des aides qu'à celui des règles d'éligibilité des dépenses et de calcul de l'aide LEADER qui peut être sollicitée. Concernant l'axe 4 en particulier, ces coûts spécifiques relatifs à la connaissance des procédures dépendent de la formation reçue par les animateurs. De ce point de vue, la situation observée a été très variable. Seuls deux animateurs sur les quatre enquêtés ont été formés au dispositif (la DRAAF Bourgogne proposait des journées de formation, de deux à cinq jours), et ce, deux ans après le début de la mise en ouvre du dispositif. Les animateurs ayant participé à ces formations ont jugé cette formation inutile car elle ne reprenait que les grandes lignes du règlement du dispositif, et qu'à ce moment de la mise en œuvre, ils avaient déjà connaissance de ces éléments. En définitive, les animateurs estiment n'avoir été complètement opérationnels, au mieux, qu'à partir de 6 mois, délai pouvant aller jusqu'à un an et demi pour certains d'entre eux. À cela s'ajoutent les problèmes liés à l'utilisation du logiciel OSIRIS, qui s'est révélé être difficile d'accès selon les animateurs, et sujet à de fréquents dysfonctionnements informatiques.

Ainsi, même si la situation s'est améliorée petit à petit, les coûts de transaction générés par les apprentissages spécifiques aux programmes LEADER ont été particulièrement élevés de par sa nature même (une animation dédiée étant prévue dans le cadre du programme). Dans ces conditions, un animateur ayant une expérience importante dans la gestion de fonds communautaires et ayant déjà intégré les mécanismes de fonctionnement du programme, a fait donc supporter à sa structure moins de coûts qu'un animateur n'ayant aucune expérience antérieure à la dernière génération de LEADER. Ce dernier cas est pourtant le plus fréquemment rencontré dans les enquêtes, la quasi-totalité des animateurs LEADER ne travaillent dans ce domaine que depuis le début de la dernière programmation (2008). Seule l'animatrice du GAL du projet 3 possède une antériorité, ce qui se répercute bien sur le niveau d'aide fournie (cf. le niveau élevé de la note globale d'aide du GAL projet 3) et le niveau faible de coût de transaction supporté par les animateurs ( 12,5 pour le projet 3 contre 20 à 27 pour les autres projets). Une expérience plus grande de l'animateur est donc corrélée à des coûts de transaction plus 
faibles mais également à une aide plus importante aux porteurs de projet.

\section{La nécessité de coordinations multiples pouvant élever le niveau des coûts de transaction pris en charge par l'équipe d'animation}

Les coûts de coordination peuvent être de diverses origines : avec les autres équipes d'animation, les co-financeurs, les services de l'État et dans le cas de LEADER entre GAL et structure porteuse.

La qualité des relations des animateurs du GAL avec les autres équipes d'animation apparaît comme étant un facteur important de limitation des coûts de transaction. À la question «Quelle est la qualité des relations que vous entretenez avec les autres acteurs animant également les projets? », les animateurs des Pays et GAL se positionnent tous entre 1 et 3 sur une échelle de 1 à 7 . Les relations qu'ils entretiennent sont donc plutôt bonnes. Toutefois, dans les rares cas où la structure porteuse entretient des relations difficiles, les coûts de coordination et de transaction sont élevés pour cette structure (cas du GAL concerné par le projet 4).

Les relations avec les co-financeurs constituent également une source importante de coûts de transaction. Dans les départements où le Conseil général a mis en place une ligne budgétaire spécialement dédiée à LEADER (comme l'Allier), l'ensemble des structures d'animation reconnaissent que les démarches en sont grandement facilitées. À l'inverse, dans les deux régions, les relations avec les Conseils régionaux apparaissent plus difficiles.

La coordination avec les services de l'État est source de coûts de transaction très différenciés. Ainsi, les appréciations portées par les animateurs varient-elles sur la quasi-totalité de l'échelle (de 1 à 7). Les animateurs mettent en avant divers éléments, tels que des divergences d'appréciation sur les dates de délibération sur les plans de financement des porteurs de projets publics (entre ASP et DRAAF), le manque de moyens en ingénierie. Enfin, l'entente entre GAL et structure porteuse est une condition nécessaire au bon fonctionnement du programme, sous peine d'engendrer des coûts de transaction très élevés (cas projet 4 ).

À noter que le GAL concerné par le projet 4 rencontre de grandes difficultés dans la mise en œuvre de sa stratégie à cause d'une divergence politique importante entre la présidente du GAL et celle du Pays. De plus, les communautés de communes du territoire, ont écarté la possibilité de maîtrise d'ouvrage du projet par l'une ou l'autre de ces deux dernières structures publiques, alors qu'il s'agissait de la solution la plus souhaitable. Enfin, les relations avec les autres acteurs animant les projets ne sont pas très développées tout particulièrement avec les chambres consulaires. Couplé avec une expérience faible de l'animateur de ce GAL, l'ensemble de ces difficultés a conduit à réorienter le dossier vers d'autres financements. À l'inverse, l'animatrice du GAL dans lequel a été conduit le projet 3 précise que les communautés de communes constituent pour elle le relais territorial de LEADER en l'informant des projets potentiellement éligibles au programme LEADER. Les coûts de transaction supportés par l'équipe d'animation pour ce projet sont les plus faibles $(12,5)$, compte tenu de l'ampleur du projet (130 K€ de coût total), grâce à la fois à la qualité des coordinations à l'œuvre et à l'expérience de l'animatrice.

\section{Conclusion}

Cette analyse comparée des projets LEADER dans deux régions françaises permet de pointer les conditions requises pour une traduction opérationnelle d'un dispositif et des coûts de transactions qui y sont liés au regard des avantages qu'ils présentent en termes de territorialisation. En complément des écrits théoriques sur la territorialisation des politiques publiques, ce travail de recherche appliquée à des projets locaux a cherché à tester une méthode d'évaluation des avantages et des coûts de nouvelles modalités de gouvernance territoriale. Au-delà des difficultés méthodologiques pour estimer le " prix à payer », une telle investigation permet de mettre en lumière que cette innovation territoriale génère des coûts spécifiques dont la nature et le niveau sont en partie spécifiques aux programmes LEADER, mais aussi très dépendants des conditions locales. En premier lieu, un porteur de projet privé supporte des niveaux de coûts de transaction supérieurs en raison des difficultés de montage des dossiers LEADER impliquant systématiquement un cofinancement. Ceci explique ainsi largement les difficultés du programme LEADER à soutenir les initiatives privées 
(Mathé et al., 2014). En second lieu, une qualité satisfaisante des coordinations entre le GAL, la structure porteuse, les intercommunalités, les services de l'État et des collectivités territoriales diminue largement les incertitudes supportées par les équipes d'animation, qui sont dès lors en mesure d'accompagner d'autant plus efficacement les porteurs de projets qu'ils ont une expérience avérée en matière de gestion de programmes européens. En termes de répartition des coûts de transaction, il a été montré qu'un soutien de l'équipe d'animation à tous les stades de la procédure diminue sensiblement le niveau des incertitudes, et donc de coûts de transaction supportés par les porteurs de projet.

Lorsque toutes les conditions favorables (existence de coordinations locales, expérience de l'équipe d'animation et du porteur de projet) sont réunies et se combinent positivement, les coûts de transaction peuvent être minimisés à la fois pour l'équipe d'animation et le porteur de projet. Inversement, si les mêmes facteurs se cumulent mais de façon négative (effets de compétition, surenchères, etc.), les coûts de transaction peuvent être élevés tant pour le porteur de projet que l'équipe d'animation. Une attention particulière devrait être accordée aux dispositifs de formation aux spécificités des programmes. De telles formations seraient à prévoir avant le démarrage de la mise en œuvre pour réduire les coûts de transaction supportés par tous les acteurs. Elles pourraient prendre la forme de formation-action adaptée aux nouvelles compétences à développer comme le suggèrent Lardon et al. (2008). Pour l'équipe d'animation, la sensibilité des coûts de transaction à la qualité des coordinations locales peut être atténuée en prévoyant dès le début de la programmation des enveloppes dédiées, pour éviter des longues discussions et recherches de cofinancement.

En définitive, ces résultats soulignent le rôle capital de l'ingénierie territoriale dans l'émergence des projets innovants de développement rural, dont les bénéfices en termes de territorialisation peuvent amplement justifier les coûts publics engagés, et peuvent, sous condition d'expérience de l'animateur, de bonne qualité des relations entre les catégories d'acteurs, fortement réduire les coûts de transaction supportés par les porteurs de projets. Les enseignements dégagés ici pourraient utilement être mis à profit par les Conseils régionaux qui ont désormais la responsabilité de la nouvelle programmation du programme de développement rural sur la période 2014-2020. 


\section{Annexe 1 : Questions de synthèse permettant d'apprécier le degré de territorialisation des projets} étudiés

\section{Synthesis questions used to assess the degree of territorialization of the projects studied}

\begin{tabular}{|c|c|c|}
\hline Questions (Q1 à Q 10) & Modalités & Notation retenue \\
\hline $\begin{array}{l}\text { Q } 1 \text { : Le projet répond-il à un enjeu spécifique au terri- } \\
\text { toire? }\end{array}$ & Oui/Non & Oui $=1 ;$ Non $=0$ \\
\hline $\begin{array}{l}\text { Q2 : Le projet vise-t-il à valoriser des ressources spéci- } \\
\text { fiques au territoire? }\end{array}$ & Oui/Non & Oui $=1 ;$ Non $=0$ \\
\hline $\begin{array}{l}\text { Q3 : Le projet valorise-t-il des Signes d'Identification de } \\
\text { la Qualité ou de l'Origine? }\end{array}$ & Oui/Non & Oui $=1 ;$ Non $=0$ \\
\hline Bilan ancrage du projet (3 questions précédentes) & & 0 à 3 maximum \\
\hline $\begin{array}{l}\text { Q4 : Le projet concerne-t-il un ou plusieurs « secteur » } \\
\text { (agricole, tourisme, social, culturel, commercial)? }\end{array}$ & $\begin{array}{l}\text { Oui }(\text { au moins } 2 \ll \text { secteurs } ») / \text { non } \\
(1 \text { seul })\end{array}$ & Oui $=1 ;$ Non $=0$ \\
\hline $\begin{array}{l}\text { Q5 : Le projet mobilise-t-il une diversité de co-finance- } \\
\text { ments (hors maître d'œuvre)? }\end{array}$ & $\begin{array}{l}\text { Oui (au moins } 2 \text { financements } \\
\text { différents)/non ( } 1 \text { seul) }\end{array}$ & Oui $=1 ;$ Non $=0$ \\
\hline $\begin{array}{l}\text { Q6 : Le projet a-t-il abouti à la création de nouveaux } \\
\text { produits ou services, nouvelles dynamiques de mise en } \\
\text { réseau? }\end{array}$ & Oui/Non & Oui $=1 ;$ Non $=0$ \\
\hline Bilan intersectorialité (3 questions précédentes) & & 0 à 3 maximum \\
\hline $\begin{array}{l}\text { Q7 : Le projet donne-t-il lieu à une organisation dédiée } \\
\text { des acteurs dans la gestion du projet (comité de pilotage, } \\
\text { groupe de travail, etc.)? }\end{array}$ & Oui/Non & Oui $=1 ;$ Non $=0$ \\
\hline $\begin{array}{l}\text { Q8 : Existe-t-il une coordination « interne » (c'est-à-dire } \\
\text { avec d'autres projets portés par le même maître d'ouvrage } \\
\text { (avant ou en même temps que le projet étudié)? }\end{array}$ & Oui/Non & Oui $=1 ;$ Non $=0$ \\
\hline $\begin{array}{l}\text { Q 9: Existe-t-il une coordination « externe » (c'est-à-dire } \\
\text { avec des projets portés par d'autres acteurs dans un cadre } \\
\text { local ou plus large)? }\end{array}$ & Oui/Non & Oui $=1 ;$ Non $=0$ \\
\hline $\begin{array}{l}\text { Q } 10 \text { : Le projet a-t-i favorisé la création de nouveaux } \\
\text { réseaux afin de le rendre plus pérenne ou efficace? }\end{array}$ & Oui/Non & Oui $=1 ;$ Non $=0$ \\
\hline Bilan coordination entre acteurs & & 0 à 4 maximum \\
\hline $\begin{array}{l}\text { Q } 11 \text { : Dans quelle mesure le projet poursuit-il des objec- } \\
\text { tifs transversaux et durables (macro ou mésoéconomique, } \\
\text { environnementaux, sociaux)? }\end{array}$ & $\begin{array}{l}\text { Objectifs d'ordre macro ou méso- } \\
\text { économique, environnementaux, } \\
\text { sociaux }\end{array}$ & $\begin{array}{l}\text { Aucun }=0 \\
1 \text { type d'objectifs }=1 \\
2 \text { types d'objectifs }=2 \\
3 \text { types d'objectifs }=3\end{array}$ \\
\hline Bilan transversalité & & 0 à 3 maximum \\
\hline
\end{tabular}




\section{Annexe 2 : Les questions posées pour l'évaluation de la spécificité des actifs et l'incertitude des projets}

\section{Questions used to evaluate projects' asset specificity and level of uncertainty}

\begin{tabular}{|c|c|c|}
\hline Variables & $\begin{array}{l}\text { Exemples de questions posées pour l'estimation des coûts de } \\
\text { transaction (échelle de Lickert) }\end{array}$ & $\begin{array}{l}\text { Exemples de questions qualitatives relatives } \\
\text { à la compréhension de la mise en oeuvre }\end{array}$ \\
\hline Vl (type d'animation) & $\begin{array}{l}\text { Quel est le nombre de personnes s'occupant de la gestion de } \\
\text { Leader? Ce(s) poste(s) sont-ils dédiés à l'animation Leader? } \\
\text { Si plusieurs personnes, que pensez-vous de la coordination } \\
\text { entre ces personnes : placez votre appréciation sur une } \\
\text { échelle de } 1 \text { à } 7,7 \text { étant coordonnation inexistante }(2)\end{array}$ & $\begin{array}{l}\text { D'autres acteurs sont-ils impliqués égale- } \\
\text { ment dans l'animation des projets? Quelles } \\
\text { sont vos relations? Que pensez-vous de la } \\
\text { coordination entre les animateurs? (2) }\end{array}$ \\
\hline $\begin{array}{l}\text { V2 (adéquation du projet } \\
\text { avec le dispositif de } \\
\text { financement) }\end{array}$ & $\begin{array}{l}\text { Pouvez-vous apprécier pour chaque structure sollicitée si } \\
\text { ce projet correspondait à ses objectifs, en estimant votre } \\
\text { appréciation sur une échelle de } 1 \text { à } 7,1 \text { étant une adéquation } \\
\text { parfaite, } 7 \text { une correspondance nulle (1) }\end{array}$ & $\begin{array}{l}\text { Comment avez-vous été mis en relation avec } \\
\text { le porteur de projet (démarchage, appel, } \\
\text { etc)? (1) }\end{array}$ \\
\hline $\begin{array}{l}\text { V3 (turn-over des } \\
\text { équipes) }\end{array}$ & $\begin{array}{l}\text { Pour un même organisme, avez-vous eu affaire à plusieurs } \\
\text { équipes qui se sont succédé? Quelle influence cela a-t-il } \\
\text { eu sur l'aide reçue : échelle de } 1 \text { à } 7: 1 \text { : peu d'influence; } 7 \\
\text { fortement compromis (1) }\end{array}$ & $\begin{array}{l}\text { Avez-vous perçu les répercussions du chan- } \\
\text { gement d'équipe sur l'animation du GAL? } \\
\text { sur le suivi de votre dossier? (1) }\end{array}$ \\
\hline $\begin{array}{l}\text { V4 (expérience des por- } \\
\text { teurs de projet) }\end{array}$ & $\begin{array}{l}\text { De manière générale, que vous a apporté votre expérience } \\
\text { passée dans le montage de projet, si vous en possédez } \\
\text { une? Vous a-t-elle aidés dans la réalisation de votre projet } \\
\text { actuel?échelle de } 1 \text { (expérience m’a beaucoup aidé; } 7 \text { : expé- } \\
\text { rience m’a peu servi) }\end{array}$ & \\
\hline $\begin{array}{l}\text { V5 (expérience des } \\
\text { membres de l'équipe } \\
\text { d'animation) }\end{array}$ & $\begin{array}{l}\text { Pouvez-vous estimer le temps qu'il vous a fallu pour être } \\
\text { opérationnel dans la structure? Quel impact a eu le renou- } \\
\text { vellement du PRDH? Diriez-vous que ce changement a pour } \\
\text { vous un impact inexistant, faible, plutôt faible, important } \\
\text { (échelle } 1 \text { à } 7 \text { )? }\end{array}$ & \\
\hline $\begin{array}{l}\text { V6 (nature juridique } \\
\text { porteur de projet) }\end{array}$ & & $\begin{array}{l}\text { De manière générale, suivez-vous de façon } \\
\text { régulière certains porteurs de projet qui ont } \\
\text { des caractéristiques particulières? pourquoi? }\end{array}$ \\
\hline $\begin{array}{l}\text { V7 (nature des relations } \\
\text { GAL- territoires de } \\
\text { projets) }\end{array}$ & $\begin{array}{l}\text { Pourriez-vous placer votre appréciation sur une échelle de } \\
1 \text { à } 7 \text { ( } 7 \text { étant relations exécrables, } 1 \text { de très bonnes relations) } \\
\text { en ce qui concerne les relations entre les équipes d'anima- } \\
\text { tion? (2) }\end{array}$ & \\
\hline $\begin{array}{l}\text { V8 (nature des relations } \\
\text { entre GAL, services } \\
\text { de l'État, territoires de } \\
\text { projet) }\end{array}$ & $\begin{array}{l}\text { Avez-vous le sentiment d'être aidé par les différents services } \\
\text { de l'État? Comment jugez-vous l'implication des services } \\
\text { de l'État sur une échelle de } 1 \text { à } 7 \text { ( } 1 \text { étant une implication } \\
\text { maximum) (2) }\end{array}$ & \\
\hline $\begin{array}{l}\text { V9 (antériorité des } \\
\text { relations porteurs de } \\
\text { projet, GAL, territoires } \\
\text { de projet) }\end{array}$ & $\begin{array}{l}\text { Avez-vous déjà bénéficié de soutien dans le passé? Dans } \\
\text { quelle mesure ces connaissances vous ont aidé pour la réa- } \\
\text { lisation du projet actuel? (réponse sur échelle de } 1 \text { à } 7: 1= \\
\text { connaissances m’ont beaucoup aidé) (1) }\end{array}$ & \\
\hline V10 Délais de paiement & $\begin{array}{l}\text { Avez-vous dû faire face à des retards dans les paiements des } \\
\text { différentes subventions qui vous ont été accordées? (1) } \\
\text { Si oui, coment ce retard vous a-t-il affecté? échelle de } 1 \text { à } 7 \\
\text { (1 : pas d'impact sur activité et santé financière } 7 \text { : grandes } \\
\text { difficultés pour poursuivre activité) } \\
\text { Sinon, comment jugez-vous la possibilité que cela arrive? } \\
\text { (1 probabilité faible, } 7 \text { : probabilité forte) }\end{array}$ & $\begin{array}{l}\text { Avez-vous pu régler les problèmes de délai } \\
\text { rapidement? Quelles démarches avez-vous } \\
\text { entamé? }\end{array}$ \\
\hline $\begin{array}{l}\text { V11 (Pérennité du por- } \\
\text { teur de projet) }\end{array}$ & $\begin{array}{l}\text { Comment jugez-vous la pérennité du porteur de projet? Sur } \\
\text { quels élements vous basez-vous pour répondre à cette ques- } \\
\text { tion? Pouvez-vous apprécier ce critère sur une échelle de } 1 \text { à } \\
7 \text { ( } 1 \text { étant une pérennité importante) (2) }\end{array}$ & \\
\hline
\end{tabular}

(1) Questionnaire porteur de projet

(2) Questionnaire équipe d'animation 


\section{Annexe 3 : Bilan du degré de territorialisation des 18 projets Summary of the degree of territorialization of the 18 projects studied}

Quelques caractéristiques des projets étudiés de façon approfondie Selected characteristics of the projects analyzed in detail

\begin{tabular}{|l|c|c|c|c|}
\hline \multicolumn{1}{|c|}{ Projets } & Nature & Maître d'ouvrage (porteur) & $\begin{array}{c}\text { Localisation } \\
\text { (département) }\end{array}$ & $\begin{array}{c}\text { Existence et nature du } \\
\text { réseau (réponse Q10) }\end{array}$ \\
\hline Pul* & $\begin{array}{c}\text { Offre accueil qualifié } \\
\text { jeunes dans château }\end{array}$ & $\begin{array}{c}\text { Communauté de com- } \\
\text { munes }\end{array}$ & 03 & 1 \\
\hline $1^{*}$ & $\begin{array}{c}\text { Communication collectifs } \\
\text { agriculteurs }\end{array}$ & Association agriculteurs & 43 & 1 \\
\hline $\mathrm{A}^{*}$ & Création caveau collectif & Association défense AOP & 71 & 0 \\
\hline $\operatorname{Pr} 1^{*}$ & $\begin{array}{c}\text { Création établissement } \\
\text { appui créateurs entreprises }\end{array}$ & SARL & O3 & 1 \\
\hline
\end{tabular}

Estimation du degré de territorialisation des projets étudiés Estimation of the degree of territorialization of the projects studied

\begin{tabular}{|c|c|c|c|c|c|c|c|c|c|c|c|c|c|c|c|c|c|c|}
\hline \multirow[b]{2}{*}{ Projets } & \multicolumn{8}{|c|}{ Porteurs de projet public } & \multicolumn{5}{|c|}{$\begin{array}{l}\text { Porteurs de projet } \\
\text { associatifs }\end{array}$} & \multicolumn{5}{|c|}{ Porteurs de projet privés } \\
\hline & $\mathrm{Pul}^{*}$ & Pu2 & Pu3 & $\mathrm{Pu} 4$ & Pu 5 & Pu6 & $\mathrm{Pu} 7$ & Pu8 & $\mathrm{Al}^{*}$ & $\mathrm{~A} 2^{*}$ & A3 & A4 & A5 & $\operatorname{Pr} 1^{*}$ & Pr2 & $\operatorname{Pr} 3$ & $\operatorname{Pr} 4$ & $\operatorname{Pr} 5$ \\
\hline $\begin{array}{l}\text { Degré d'ancrage } \\
\text { territorial }\end{array}$ & 2 & 3 & 2 & 1 & 1 & 1 & 1 & 3 & 2 & 2 & 0 & 0 & 1 & 2 & 2 & 2 & 2 & 1 \\
\hline $\begin{array}{l}\text { Degré } \\
\text { d'intersec-torialité }\end{array}$ & 3 & 3 & 3 & 2 & 3 & 3 & 3 & 3 & 3 & 2 & 1 & 2 & 2 & 3 & 2 & 2 & 1 & 1 \\
\hline $\begin{array}{l}\text { Degré de } \\
\text { coordination entre } \\
\text { acteurs }\end{array}$ & 4 & 3 & 4 & 2 & 4 & 3 & 3 & 2 & 2 & 3 & 2 & 4 & 4 & 3 & 3 & 2 & 2 & 1 \\
\hline $\begin{array}{l}\text { Degré de } \\
\text { transversalité }\end{array}$ & 3 & 3 & 3 & 2 & 3 & 3 & 3 & 2 & 2 & 2 & 1 & 1 & 1 & 2 & 2 & 2 & 3 & 2 \\
\hline $\begin{array}{l}\text { Degré de } \\
\text { territorialisation }\end{array}$ & 12 & 12 & 12 & 7 & 11 & 10 & 10 & 8 & 9 & 9 & 4 & 8 & 8 & 10 & 9 & 8 & 8 & 5 \\
\hline
\end{tabular}

*: Projets retenus dans l'article

\section{Bibliographie}

Balny P., Bobenriether O., Martin de Lagarde O., 2010. Rapport de mise en cuvre des axes 3 et 4 du FEADER. Rapport CGAAER no 2091, Ministère de l'Agriculture, 250 p., (non publié).

Barbarot A.-S., Renard T., Vollet D., Lepicier D., 2013. Propositions méthodologiques pour mesurer la territorialisation des projets de développement rural, Économie Rurale, vol. 336, no 4, p. 81-88.

Berriet-Solliec M., Chabe-Ferret S. (dir.), 2011 . Analyse des politiques de développement rural en Auvergne et en Bourgogne, pour un pilotage efficace de l'action régionale, Rapport scientifique final, PSDR 3 2007-11, 44 p., (non publié).

Berriet-Solliec M., Trouve A., 2013. Développement des politiques de projet. Quels enjeux pour les politiques de développement rural?, Économie Rurale, vol. 335, n 3, p. 6-18.

Berriet-Solliec M., Delorme H., Laurent C., Mundler P., Perraud D., 2006. Regulation of agriculture: the Regions as a new locus for working towards territorial coherence between agricultural policies? The Rhône-Alpes region in the European context, Canadian Journal of regional science, $n^{\circ} 29$, p. 55-68.

Chabé-Ferret S., Subervie J., 2013. How much green for the buck? estimating additional and windfall effects of French agro-environmental schemes by DID matching, Journal of Environmental Economics and Management, p. 12-27.

Chevalier P., Dedeire M., 2014. Application du programme LEADER selon les principes du développement local, Économie rurale, vol. 342, n 4, p. 274-290. 
Chia E., Torre A., Rey-Valette H., 2008. Vers une «technologie » de la gouvernance territoriale !, Norois, n 209 , p. 167-177.

Coase R., 2005. L'entreprise, le marche et le droit, Paris, Éditions d'Organisation, $243 \mathrm{p}$.

Dahlman C., 1979. The problem of externality. The journal of Law and Economics, p. 141-162.

Colletis-Wahl K., Pecqueur B., 2001. Territories, Development and Specific Resources: what analytical framework? Regional Studies, vol. 35, no 5, p. 449-459.

Cour des Comptes Européennes, 2010. Mise en ceuvre de l'approche LEADER pour le développement rural, Luxembourg, Office des publications de IUnion Européenne, 150 p., (non publié).

Dargan L, Shusksmith M., 2008. LEADER and innovation, Sociologia Ruralis, vol 3, n 48, p. 274-291.

Dax T., Strahl W., Kirwan J., Maye D., 2016. The Leader programme 2007-2013: Enabling or disabling social innovation and neo-endogenous development? Insights from Austria and Ireland, European Urban and Regional Studies, vol. 23, $n^{\circ}$ 1, p. 56-68.

Gloria M., 2011. Territorialisation des politiques de développement rural. Analyse comparée de la valeur ajoutée territoriale du programme LEADER, Mémoire d'ingénieur, AgroSup Dijon, $102 \mathrm{p}$.

Irwin Eg., Isserman Am., Kilkenny M., Partridge M.D., 2010. A century of Research on Rural Development and Regional Issues, American Journal of Agricultural Economics, vol 2, no 92, p. 522-533.

Képhaliacos C., Robin P., 2004. Implementing environmental quality standards via collective projects in the French CTE procedure, International Journal of Agricultural Resources, Governance and Ecology, n ${ }^{\circ}$ 3, p. 2-10.

Lardon S., Chia E., Rey-Valette H., 2008. Dispositifs et outils de gouvernance territoriale, Norois, no 209, p. 7-13.

Mac Cann L., Colby K.W., Easter A., Kastermine A., KupeRAN K., 2005. Transaction Cost Measurement for evaluating environmental policies, Ecological Economies, $n^{\circ}$ 52, p. 527-542.

Mathé J., Vollet D., Lépicier, D., Le Roy A., 2014. Évaluation régionale de LEADER en Auvergne, un bilan mitigé et contrasté en termes de valeur ajoutée, Sciences, Eaux et Territoires, $\mathrm{n}^{\circ} 13$, p. 38-43.

Maurel M. C., Chevalier P., Lacquement G., 2014. Transfert et apprentissage du modèle LEADER en Europe centrale, Paris, L'Harmattan, 300 p.

Measson L., 2007. L'efficacité territoriale et l'évaluation : penser le déploiement spatial du politique grâce au programme européen $L E A D E R$. Thèse de doctorat en géographie, Université Joseph Fourier Grenoble I, 506 p.

Menard C., 2003. L'approche néo-institutionnelle : des concepts, une méthode, des résultats, Cahiers d'économie politique, $\mathrm{n}^{\circ} 44$, p. 103-118.

Michel S., 2010. Analyse comparative des modalités de mise en ouvre des axes 3 et 4 du PDRH en Awvergne et en Bourgogne. Mémoire d'ingénieur, AgroSupDijon, 83 p.
Observatoire Européen LEADER,1999. Évaluer la valeur ajoutée de l'approche LEADER, Innovation en milieu rural, Cahier $n^{\circ} 4$ de l'association Européenne pour l'Information sur le Développement Local (AEIDL), Bruxelles, 20 p.

OCDE, 2007. Les coûts de mise en œuvre des politiques agricoles, OCDE, Paris, 14 p.

Perrin C., 2011. La territorialisation de l'agriculture périurbaine du Chianti : entre terroir et proximité urbaine, Norois, 221 , p. 97-109.

Rey-Valette H., Mathé S., 2012. L'évaluation de la gouvernance territoriale, enjeux et propositions méthodologiques, Revue d'Économie Régionale et Urbaine, nº 5, p. 783-804.

Rousset S., Louis M., 2012. Coûts de transaction et adoption des MAE territorialisées à enjeu eau, Revue d'Économie Régionale \& Urbaine, $n^{\circ}$ 1, p. 65-89.

Sanchez-Zamora P., Lollardo-Cobos R., Cena-Delgado F., 2014. Rural areas face the economic crisis: Analyzing the determinants of successful territorial dynamics, Journal of Rural Studies, no 35, p. 11-25.

Strzelecka M., 2015. The Prospects for Empowerment through Local Governance for Tourism: the LEADER Approach, Journal of Rural \& Community Development, vol. 10, n 3, p. 78-97.

Tiveyrat D., 2011 . La territorialisation des politiques de développement rural. Proposition d'une méthode de calcul du degré de territorialisation des projets axe 3 LEADER et hors LEADER. Comparatif infrarégional Awvergne et Bourgogne, Mémoire de Master 1, Université Blaise-Pascal de Clermont-Ferrand, $82 \mathrm{p}$.

Thuessen A.A., Nielsen N.C., 2014. A Territorial Perspective on Eu'S Leader Approach In Denmark: The Added Value Of Community-Led Local Development Of Rural And Coastal Areas In A Multi-Level Governance Settings, European Countryside, vol. 6, n 4, p. 307-326.

Trouvé A., Berriet-Solliec M., Déprés C., 2007. Charting and theorizing the territorialisation of agricultural policy. Journal of Rural Studies, Vol. 4, no 23, p. 443-452.

Valqui-Vidal R.-V., 2009, Community facilitation of problem structuring and decision making processes: Experiences from the EU LEADER+ program, European Journal of Operational Research, $\mathrm{n}^{\circ} 199$, p. 803-810.

Vollet D., Callois J-M., Aznar O., Yelkouni M., 2010. Coûts de transaction et politiques agri-environnementales, Revue Canadienne d'évaluation de programme, vol. 24 n 2, p. 103130.

Zahm F., Kephaliacos A., Vernier F., Del Corso J.-P., Kuentz T., 2016, Évaluation intégrée des mesures agroenvironnementales territorialisées à enjeu « qualité des eaux » sur la période 2007 à 2011 : le projet MAEVEAU, in Rousset S., Zанм F., 2016. Eaux et Territoires agricoles : dépasser les contradictions? Résultats de sept projets de recherche, Commissariat Général au Développement Durable, p. 61-70.

Williamson O., 1994, Les institutions de l'économie, Paris, InterEditions, $404 \mathrm{p}$. 